\section{Isolation of vaccinia MVA recombinants using the viral F13L gene as the selective marker}

\author{
Juana M. Sánchez-Puig and Rafael Blasco \\ INIA, Madrid, Spain
}

BioTechniques 39:665-674 (November 2005)

doi 10.2144/000112012

Modified vaccinia Ankara (MVA) is a highly attenuated vaccine vector that has an excellent vaccine safety record. Also, as a eukaryotic gene expression vector, MVA can be used in a biosafety level 1 setup, in contrast to more virulent vaccinia virus strains. Isolation of recombinant MVA involves repeated plaquing of the virus and is burdensome because virus plaques are slow to develop and difficult to recognize. To facilitate the generation of MVA recombinants, we have developed a cloning system for MVA based on the selection of the viral F13L gene. Deletion of F13L in MVA produced a small plaque phenotype and a reduction in extracellular virus formation, indicating a severe block in cell-to-cell spread. When using the $F 13 L$ knockout virus as the parental virus, reintroduction of the F13L gene in the original locus was used as an efficient selection for the isolation of virus recombinants. The selection procedure can be done entirely in the permissive baby hamster kidney (BHK)-21 cell line, does not require plaque isolation, and rendered close to $100 \%$ recombinant virus.

\section{INTRODUCTION}

Vaccinia viruses are robust eukaryotic expression vectors that have been used for a number of different studies in biochemistry, cell biology, and immunology. Poxviruses, which include vaccinia virus, have several advantages for use as expression or vaccine vectors, including their large insertion capacity, their cytoplasmic site of replication, and the relatively high expression levels and proper posttranslational modifications of foreign proteins.

Most vaccinia virus strains, such as the ones used in the smallpox eradication campaign, readily replicate in human cells and have been associated with a range of clinical complications in vaccinees. This fact is presently considered a major problem for the use of these strains as recombinant vaccines for mass vaccination. For laboratory use, in addition to mandatory biosafety level 2 procedures, proper precautions must be taken to prevent accidental exposure when managing replicationcompetent vaccinia strains.

To circumvent the problems associated with "classical" vaccinia virus strains, several laboratories have been increasingly involved in the use of more attenuated, host-restricted virus strains. Among these, modified vaccinia Ankara (MVA) has elicited considerable interest because of its excellent safety record in humans (1-4). In addition to being a promising vector for the construction of poxvirusbased recombinant vaccines, one appealing application of MVA is its use as an expression vector that can be used under biosafety level 1 laboratory conditions.

Isolation of MVA recombinants is usually more difficult and takes more time than isolation of recombinants based on other vaccinia strains. First, because of the limited virus host range, virus plaquing and amplification has to be done in primary chick embryo fibroblast (CEF) cultures or in the baby hamster kidney (BHK)-21 susceptible cell line. Second, virus plaques are difficult to visualize by vital staining and, as a consequence, alternative methods such as immunostaining of the monolayers are required.

Several marker genes, such as $\beta$ galactosidase, $\beta$-glucuronidase, and Escherichia coli gpt have been used in recombinant MVA technology (5-8). More recently, a system based on the transient incorporation of a host range gene $(\mathrm{K} 1 \mathrm{~L})$ into the MVA genome was developed (9-11). The K1L gene allows the virus to multiply in rabbit kidney RK13 cells (12), thus allowing the efficient removal of parental virus by passaging the virus in RK13 cells. With this system, the final steps in recombinant MVA isolation involve the removal of the K1L gene, a process carried out in susceptible cells in the absence of selection (11). Similarly, the viral gene E3L can be used as a selective genetic marker because expression of $\mathrm{E} 3 \mathrm{~L}$ is required for growth in CEF (13).

We have adapted a selection system based on virus plaque formation originally designed for vaccinia virus (strain WR) recombinants (14). The system takes advantage of the nonplaquing phenotype of vaccinia virus F13L deletion mutants $(15,16)$. The insertion of the foreign gene is achieved along with the reintroduction of gene F13L using the restoration of the plaquing phenotype as the selective condition. Virus recombinants isolated by F13L selection have the original MVA genetic background, making it unnecessary to eliminate the selective marker gene.

\section{MATERIALS AND METHODS}

\section{Cells, Virus, and Plasmids}

BHK-21 cells were obtained from the ATCC (ATCC-CCL10) and were grown in BHK medium (Invitrogen, Carlsbad, CA, USA) containing 5\% fetal bovine serum (FBS), $3 \mathrm{~g} / \mathrm{mL}$ tryptose phosphate broth, and $0.01 \mathrm{M}$ HEPES. The cells were mantained in a $5 \% \mathrm{CO}_{2}$ atmosphere at $37^{\circ} \mathrm{C}$. The infection/transfection protocol, plaque purification, and virus amplifications were carried out as previously described $(17,18)$. Plasmid pRBdsRed is a derivative of pRB21 (14), which contains a functional F13L, flanking sequences, and the dsRed gene downstream of a synthetic vaccinia early/late promoter.

\section{Fluorescence Microscopy}

Cells expressing autofluorescent proteins were visualized under a Nikon Eclipse TE-200E inverted 
microscope (Nikon, Tokyo, Japan) provided with a $100 \mathrm{~W}$ mercury lamp. Green fluorescent protein (GFP) fluorescence was detected using a fluorescein isothiocyanate (FITC) filter set (excitation, 465-495 nm; dicroic mirror, $505 \mathrm{~nm}$; and emission, 515-555 $\mathrm{nm})$. dsRed fluorescence was detected using a tetramethyl rhodamine isothiocyanate (TRITC) filter set (excitation, 515-560 nm; dicroic mirror, $575 \mathrm{~nm}$; and emission over $590 \mathrm{~nm}$ ). Digital photographs were captured using either a monochrome ST-7 camera (Santa Barbara Instrument Group, Santa Barbara, CA USA) or a Retiga Exi 12-bit monochrome digital camera (Qimaging, Burnaby, BC, Canada).

\section{Plasmid Construction}

Plasmid pMVARed was constructed from plasmid pRBdsRed (containing the dsRed2-N1 gene from BD Biosciences Clontech, Mountain View, CA, USA) by replacing the sequences derived from vaccinia WR (including F13L gene and flanking sequences) by F13L flanking sequences from MVA. First, a fragment containing left-flanking sequences of the F13L gene was amplified by PCR from the MVA genome using primers $5^{\prime}$-GGG GCATGCGATAAAGTTTCGAAAC AGCAAA-3' (SphI site underlined) and $5^{\prime}$-CATTTTGCTCGAGCAGGT ACCGGTGCAA-3' (XhoI site underlined). After $S p h \mathrm{I} / \mathrm{XhoI}$ digestion, the fragment was inserted into the corresponding sites in plasmid pRBdsRed to generate $\mathrm{pMVA}_{\mathrm{L}}-\mathrm{Red}$. Then, a fragment containing a right-flanking sequence of $\mathrm{F} 13 \mathrm{~L}$ was amplified by PCR from the MVA genome using primers 5'-GAGAGAGCTCGGGT ATCTAGCCACAGTA-3' (SacI site underlined) and $5^{\prime}$-GCGCCCACAA TGCATCTCTAGATATGTA-3' (Nsi site underlined), digested with $\mathrm{SacI}$ and NsiI, and inserted between the SacINsiI sites of plasmid $\mathrm{pMVA}_{\mathrm{L}}-\mathrm{Red}$. The resulting plasmid, pMVA-Red, contains a dsRed cassette flanked by MVA F13L flanking sequences.

Plasmid pMVA-rsGFP, designed to mediate the insertion of $F 13 \mathrm{~L}$ and a red-shifted GFP (rsGFP; Quantum Biotechnologies, Montreal, Canada) cassette into the F13L locus of vaccinia
MVA was constructed as follows. pMVA-p37-Red was constructed by replacing the left F13L flank in pMVARed by a fragment containing the left F13L flank and the complete F13L gene. This fragment was amplified by PCR from MVA DNA using oligonucleotides 5'-GGGGCATGCGATAA AGTTTCGAAACAGCAAA-3' (SphI site underlined) and $5^{\prime}$-CCCCTCGA GTTATATCTTAAGACTCTTTGAA TGTGAGCTCACCCAATCTCTCTC AAATATTTTTTTAGC-3' (XhoI site underlined). Finally, the dsRed cassette in pMVA-p37-Red was replaced with an $\mathrm{XhoI} /$ Bam HI fragment containing a rsGFP gene cassette derived from $\mathrm{pRB}$ rsGFP (19) to produce pMVA-rsGFP.

pMVA- $\beta$ Gus was constructed by replacing the rsGFP cassette with a fragment containing a polylinker, the sequence of the $\beta$-glucuronidase gene, and a synthetic vaccinia virus early/late promoter from plasmid pRB- $\beta$ Gus (20). This was achieved by cloning an XhoI/BamHI fragment from plasmid $\mathrm{pRB}-\beta \mathrm{Gus}$ into the XhoI/BamHI sites of plasmid pMVA-rsGFP.

\section{Isolation of F13L Deletion Mutant}

MVA $\triangle F 13 L$ was isolated through recombination of pMVA-Red into the MVA genome. Briefly, $1 \times 10^{6} \mathrm{BHK}-$ 21 cells were infected with vaccinia virus MVA in fresh BHK medium containing $2 \%$ fetal calf serum (FCS) at a multiplicity of infection (MOI) of 0.05 plaque-forming units (pfus) per cell and were transfected with $2 \mu \mathrm{g}$ of the plasmid pMVARed using FuGENE ${ }^{\circledR} 6$ Transfection Reagent (Roche Applied Science, Indianapolis, IN, USA). Dilutions of progeny virus were used to infect BHK21 monolayers in 6-well plates that were maintained at $37^{\circ} \mathrm{C}$ for 3 days under an agarose overlay. Plaque-forming viruses were selected by plaquing the progeny under an agarose overlay. Following a 3-day incubation, large plaques were selected. After a total of three rounds of plaquing, virus stock was cloned by limit dilution in BHK-21 monolayers in 96-well plates. Small plaques showing red fluorescence were harvested and amplified by five cycles of infection in BHK-21 cells.

\section{Isolation of MVA Recombinants}

Monolayers of approximately $10^{6}$ BHK cells in 6-well plates were infected with MVA $\triangle \mathrm{F} 13 \mathrm{~L}$ at a MOI of $0.05 \mathrm{pfu} / \mathrm{cell}$. At $1 \mathrm{~h}$ postinfection, the virus inoculum was removed, and the cells were transfected with 2 $\mu \mathrm{g}$ of a plasmid containing the F13L gene (pMVA-rsGFP or pMVA- $\beta$ Gus)
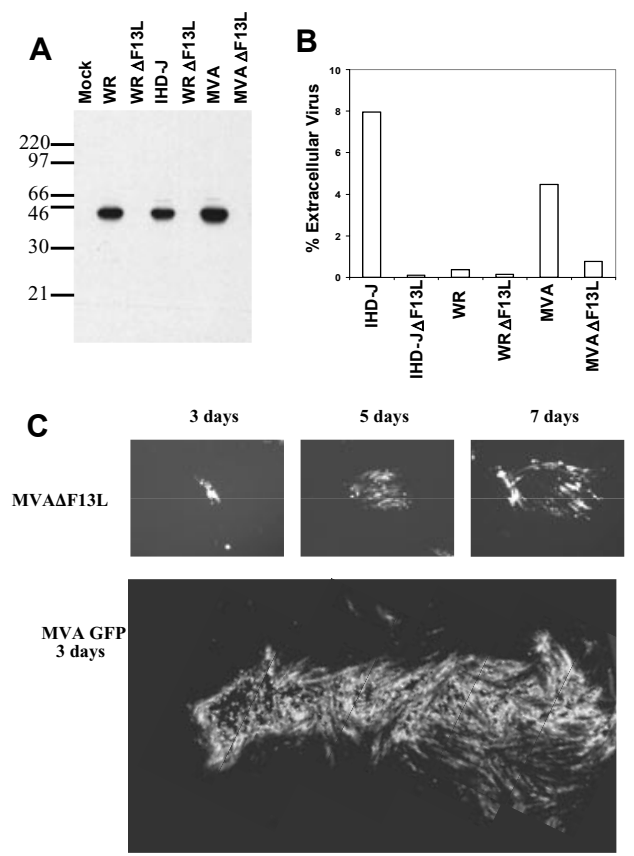

Figure 1. Characterization of MVA $\Delta F 13 L$. (A) Western blot. BHK-21 cells mock-infected or infected with the vaccinia viruses indicated in the upper part were lysed and subjected to Western blot analysis with anti-F13L monoclonal antibody. The position of the protein markers is indicated on the left. (B) Extracellular virus formation. BHK-21 cells were infected at an MOI of $5 \mathrm{pfu} / \mathrm{cell}$ and harvested at $24 \mathrm{hpi}$. The percentage of infectivity in the medium with respect to total progeny virus produced by the different viruses is indicated. (C) Plaque formation by MVA $\triangle$ F13L. BHK-21 monolayers were infected with dilutions of MVA $\triangle \mathrm{F} 13 \mathrm{~L}$. Clusters of cells displaying red fluorescence were visualized in an inverted fluorescence microscope and photographed with a $10 \times$ objective at the times postinfection indicated. Below, a plaque (3 days) formed by a GFP-expressing MVA recombinant is shown for comparison. The larger panel was assembled using four overlapping photographs. BHK, baby hamster kidney; MOI, multiplicity of infection; pfu, plaque-forming unit; hpi, hours postinfection; MVA, modified vaccinia Ankara; MVA GFP, an MVA recombinant expressing green fluorescent protein. 
using FuGENE 6 transfection reagent. The cells were maintained under 2 $\mathrm{mL}$ of fresh BHK medium containing $2 \%$ FCS. After 3 days, the cells were scraped and freeze/thawed 3 times.

Enrichment in virus recombinants was carried out by repeated passaging in BHK-21 cells. Monolayers of BHK cells in six-well plates were infected with $1 / 100$ volume of the cell lysate in BHK medium containing $2 \%$ FCS.

After $1 \mathrm{~h}$ of adsorption, the inoculum was removed and replaced with $2 \mathrm{~mL}$ of fresh BHK medium containing $2 \%$ FCS. Infections were maintained for $48 \mathrm{~h}$ and then harvested for the next passage.

\section{Western Blot Analysis}

BHK-21 cells grown in 6-well plates were infected for $24 \mathrm{~h}$ and then

A
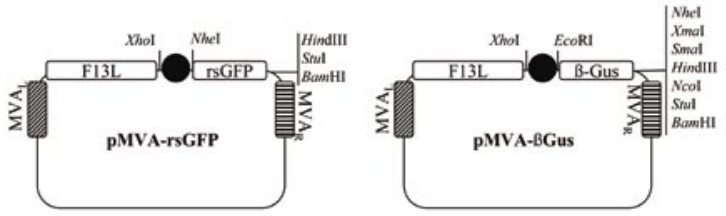

ATTTTATTTTTTTTTTTTGGAATATAAATGGCTAGCAAAGGAGAAGAACT..GFP Nhel

ATTTTATTTTTTTTTTTTGGAATATAAATAAGGAATTCTATGTTACGTCC..Gus

B

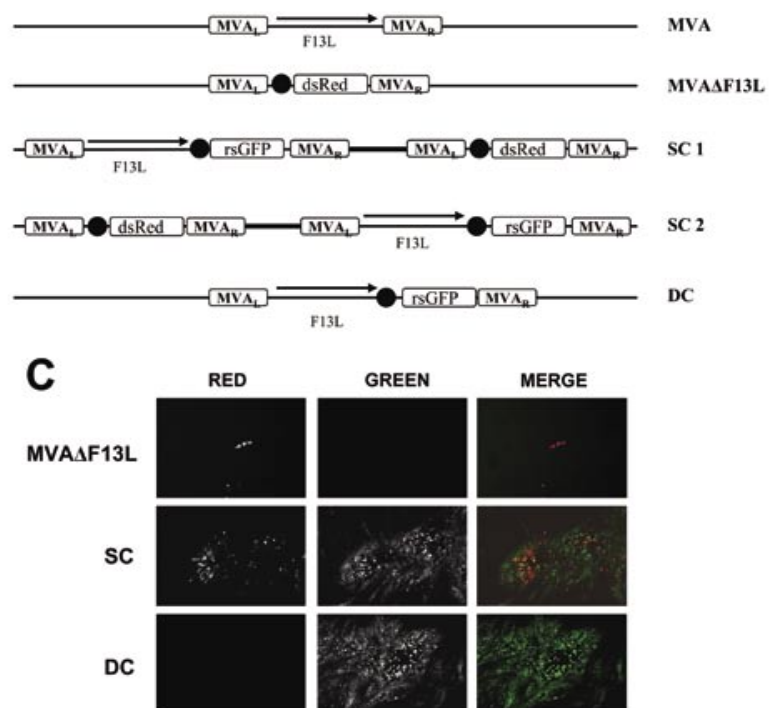

Figure 2. Isolation of MVA recombinants using F13L selection. (A) Plasmids used for selection. Plasmids pMVA-rsGFP and pMVA- $\beta$ Gus contain the GFP and $\beta$-glucuronidase gene, respectively, downstream of a vaccinia synthetic early/late promoter (black dot), together with the complete F13L gene and recombination flanks $\left(\mathrm{MVA}_{\mathrm{L}}\right.$ and $\left.\mathrm{MVA}_{\mathrm{R}}\right)$. Below, the sequence around the gene initiator codon is shown. Foreign genes can be inserted into the NheI site of pMVA-rsGFP, immediately downstream of the initiator ATG, or into the EcoRI site of pMVA- $\beta$ Gus, by placing the gene with its own ATG. (B) Representation of recombination products. The relative position of different elements in the MVA virus, the F13L MVA deletion mutant (MVA $\Delta$ F13L), and the different products obtained by recombination between MVA $\triangle \mathrm{F} 13 \mathrm{~L}$ and plasmid pMVA-rsGFP is shown. SC 1 and SC 2 are single crossover products, arising from recombination in one of the two F13L flanking sequences. $\mathrm{DC}$ is a stable double crossover, arising from recombination in the two F13L flanking sequences. (C) Identification of recombinant viruses by plaque phenotype and fluorescence. Representative plaques formed by parental virus MVA $\triangle \mathrm{F} 13 \mathrm{~L}$, single crossover recombinants (SC) and double crossover recombinants (DC) are shown as visualized for red or green fluorescence. MVA, modified vaccinia Ankara; GFP, green fluorescent protein. scraped from the

Biosciences, Piscataway, NJ, USA) in $0.05 \%$ PBS and Tween 20 containing $1 \%$ nonfat dry milk. After washing, the membranes were incubated for 1 min with a $1: 1$ mixture of solution A [2.5 mM luminol, $0.4 \mathrm{mM} p$-Coumaric acid (both from Sigma, St. Louis, MO, USA), $100 \mathrm{mM}$ Tris-HCl, $\mathrm{pH} 8.5$ ] and solution $\mathrm{B}\left(0.018 \% \mathrm{H}_{2} \mathrm{O}_{2}, 100 \mathrm{mM}\right.$ Tris- $\mathrm{HCl}, \mathrm{pH} 8.5$ ) and exposed to $\mathrm{X}$ ray film.

\section{RESULTS AND DISCUSSION} lysed in $75 \mu \mathrm{L}$ of $2 \times$ polyacrylamide gel electrophoresis (PAGE) loading buffer $[0.16 \mathrm{M}$ Tris-HCl, pH 8.8, 4\% sodium dodecyl sulfate (SDS), $10 \% \beta$-mercaptoethanol, $5 \%$ glycerol, and $0.02 \%$ bromophenol blue]. Proteins were electrophoresed in $12 \%$ SDS polyacrylamide gels and transferred to nitrocellulose membranes by electroblotting. After transfer, the membranes were incubated for 2 $\mathrm{h}$ at ambient temperature in blocking buffer [phosphatebuffered saline (PBS) containing $\quad 0.05 \%$ Tween ${ }^{\circledR} 20$ and 5\% nonfat dry milk]. The membranes were then incubated overnight at $4^{\circ} \mathrm{C}$ with monoclonal antibody anti-F13L (15B6 hybridoma supernatant, diluted 1:50 in PBS supplemented with $0.05 \%$ Tween 20 and $1 \%$ nonfat dry milk). After extensive washing with PBS- $0.05 \%$ Tween 20, the membranes were incubated for $1 \mathrm{~h}$ at room temperature with goat anti-rat or mouse immunoglobulin $\mathrm{G}$ (IgG) conjugated with horseradish peroxidase (HRP, diluted 1:3000; Amersham

\section{Isolation of MVA $\Delta \mathrm{F} 13 \mathrm{~L}$}

F13L deletion mutants in vaccinia virus strains WR and IHD-J have a strict small plaque phenotype $(15,16)$. Because MVA has a conserved F13L gene $(99.5 \%$ amino acid identity with WR F13L protein), we predicted that deletion of the F13L gene would have a similar effect in MVA. To inactivate the gene, we chose to replace most of the F13L coding sequence with a red fluorescent protein (dsRed) gene that, in addition to allowing identification of virus mutants, could facilitate the visualization of tiny plaques or even single infected cells. With this aim, we constructed plasmid pMVA-Red, which contains recombination flanks for the F13L gene and the dsRed gene under the control of a vaccinia promoter, in place of the F13L coding sequence. Recombination of this plasmid with the vaccinia MVA genome rendered virus recombinants that produced large plaques displaying red fluorescence (data not shown). One of these viruses, presumed to be the result of a single recombination event $\left(\mathrm{F}_{13} \mathrm{~L}^{+}\right.$, dsRed ${ }^{+}$; see Figure 2B), was isolated by three consecutive rounds of plaque purification in BHK-21 cells. After amplification, the virus stock was cloned by limit dilution in an attempt to detect and isolate small virus plaques that would potentially be stable double recombinants $\left(\mathrm{F} 13 \mathrm{~L}^{-}, \mathrm{dsRed}^{+}\right)$. After 3 days of infection, single wells that contained tiny fluorescent spots (fewer than 20-30 cells) and no large infected areas were selected, harvested, and amplified. Of the six clones amplified, one representative was termed MVA $\Delta \mathrm{F} 13 \mathrm{~L}$ and chosen for further characterization. 


\section{Characterization of MVA $\Delta \mathrm{F} 13 \mathrm{~L}$}

Insertion of the marker dsRed gene into the F13L locus was confirmed by PCR (data not shown). Western blot analysis with antibody to F13L confirmed the absence of the F13L protein in MVA $\Delta$ F13L-infected cells. The lack of staining was not due to strain differences because MVA F13L protein reacted with the antibody, as revealed by the presence of a protein with the expected electrophoretic mobility in extracts from cells infected with the parental MVA (Figure 1A).

In order to study the effect of the F13L deletion on MVA, we determined extracellular virus formation and plaque formation by MVA $\Delta \mathrm{F} 13 \mathrm{~L}$. Similarly to other vaccinia strains, F13L deletion resulted in a dramatic effect on plaque formation because only tiny virus plaques (detected as clusters of cells showing red fluorescence) were seen even after prolonged incubation (Figure 1C). In contrast, larger, comet-shaped plaques were visible at 2-3 days postinfection under the microscope when infecting with a F13L+ MVA virus expressing GFP. F13L deletion also affected extracellular virus formation because virus infectivity recovered in the medium of MVA $\Delta$ F13L-infected BHK-21 cells was reduced almost 6-fold compared with parental MVA (Figure 1B). These results are in agreement with observations reported for vaccinia strains WR and IHD-J and indicate that F13L deletion results in a block of enveloped virus release and cell-to-cell transmission.

\section{Design of an F13L Cloning System for MVA}

The phenotype of MVA $\triangle \mathrm{F} 13 \mathrm{~L}$ suggested that F13L could be used as an efficient selective marker for the isolation of MVA recombinants. Thus, we constructed a plasmid that incorporates the F13L gene and flanking sequences to mediate restoration of the gene into its original locus by homologous recombination. In addition, a synthetic early/late vaccinia promoter was placed downstream of the F13L gene to achieve expression of foreign genes. Plasmids pMVA-rsGFP and
pMVA- $\beta$ Gus incorporate marker genes GFP and $\beta$-glucuronidase, respectively, downstream of the vaccinia promoter (Figure 2A). Plasmid pMVArsGFP has an NheI site immediately downstream of the initiator ATG, and thus can be used to express in-frame open reading frames. Alternatively, the $E c o$ RI site in pMVA- $\beta$ Gus can be used to clone complete open reading frames including their initiator ATG.

\section{Isolation of Recombinant MVA Using F13L Selection}

To monitor the selection process, we made use of GFP as the foreign gene to be inserted into MVA. Thus, virus $\mathrm{MVA} \triangle \mathrm{F} 13 \mathrm{~L}$ and plasmid $\mathrm{pMVA-}$ rsGFP were employed for infection/ transfection experiments. Because parental virus expressed dsRed and the plasmid contained a GFP cassette, viruses harboring different recombination products (see Figure 2B) could be visualized during virus passages by inspection under the fluorescence microscope (Figure 2C). As mentioned above, parental MVA $\Delta \mathrm{F} 13 \mathrm{~L}$ virus appeared as small infected foci displaying only red fluorescence. In contrast, recombination products could be easily identified because of their larger plaque size and green fluorescence. The use of two fluorescent markers also facilitated to distinguish the viruses arising from single recombination events and the more stable "double recombinants" because the former retained both green and red fluorescence whereas the double recombinants only displayed GFP fluorescence.

Plasmid pMVA-rsGFP was transfected into cells previously infected with MVA $\triangle$ F13L to allow for recombination of the plasmid and the viral genome to occur. To test the efficiency of F13L selection, five consecutive virus passages were carried out in BHK-21 cells, and the titer of single and double recombinants after each passage was determined (Figure 3). Parallel experiments were performed using either 1/100th of the total progeny virus or 1/10th of the extracellular virus as the inoculum for the next round of infection, with similar results. During passages, infected cultures were maintained for 2-3 days, until cytopathic effect was evident, and then used for the next round of infection. Finally, to determine the composition of the virus population after each round, samples from each of the passages were diluted, plaqued on fresh monolayers of BHK-21 cells, and inspected by fluorescence microscopy. The starting virus population (virus progeny from the initial infection-transfection) contained approximately $0.1 \%$ virus recombinants. Passages increased the titer of virus recombinants, which outgrew the parental virus after the second passage. After the third passage, it was not possible to accurately determine the titer of parental virus due to the high titer of single and double recombinants. After four or five passages, the virus population consisted almost exclusively in virus recombinants, of which the overwhelming majority were double recombinants. These results indicate that the selection procedure is efficient and can be completed in 10-14 days.

The above experiment was carried out with an easily recognizable marker protein (GFP) that can be followed during the infection. To test

\begin{tabular}{|c|c|c|c|c|}
\hline Passage & Parental & SC & DC & $\% \mathrm{DC}$ \\
\hline 0 & $3.8 \times 10^{5}$ & $7.0 \times 10^{2}$ & $3.0 \times 10^{2}$ & 0.1 \\
\hline 1 & $5.1 \times 10^{6}$ & $4.0 \times 10^{4}$ & $1.5 \times 10^{5}$ & 2.8 \\
\hline 2 & $1.3 \times 10^{6}$ & $1.7 \times 10^{5}$ & $6.0 \times 10^{6}$ & 80.3 \\
\hline 3 & $2.0 \times 10^{5}$ & $1.2 \times 10^{5}$ & $3.6 \times 10^{6}$ & 91.6 \\
\hline 4 & $6.0 \times 10^{4}$ & $9.0 \times 10^{4}$ & $3.9 \times 10^{7}$ & 99.6 \\
\hline & $5.0 \times 10^{3}$ & $7.0 \times 10^{3}$ & $6.0 \times 10^{6}$ & 99.8 \\
\hline
\end{tabular}

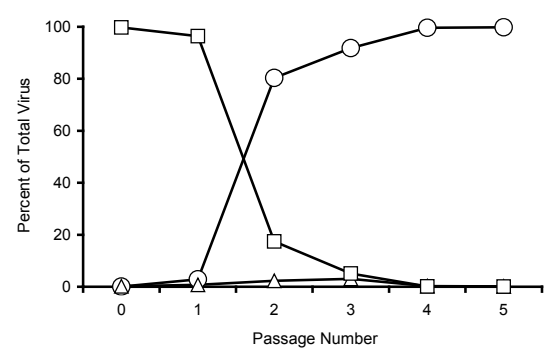

Figure 3. Enrichment in virus recombinants during serial passages. Progeny virus from passages in BHK-21 cells was analyzed by plaquing. Passage " 0 " indicates the progeny virus from the infection/transfection. Subsequent passages were carried out with 1/100th of the progeny from the previous passage. Different viruses were identified by their phenotypes: parental virus produces small infection foci showing red fluorescence, single crossover viruses (SC) form large plaques showing red and green fluorescence, and double crossover virus (DC) form large plaques showing green (but not red) fluorescence. Lower panel: the percentage of parental (squares), SC (triangles), and DC (circles) virus in the total virus population after each passage is represented. 
F13L selection without monitoring foreign gene expression, we repeated the selection procedure using the $\beta$ glucuronidase gene. To blindly test the procedure, the passages were performed without applying $\beta$-Gus staining. After selection, dsRed-negative viruses from passages 4 and 5 were cloned by limit dilution, expanded, and checked for $\beta$-glucuronidase activity. In this experiment, 12 of 12 virus clones expressed $\beta$-glucuronidase (data not shown), confirming that the selection procedure described here can be done without monitoring for foreign gene expression and further confirms that 4-5 passages in BHK-21 cells result in a high proportion of virus recombinants.

The above results illustrate the usefulness of the F13L selection system. When using classical vaccinia methods, isolation of recombinant MVA is more difficult than with other widely used vaccinia virus strains because of its strict host restriction, slower growth, and poor plaquing. To facilitate the process, several selection methods have been described. Of these, the most efficient rely on the introduction of host range genes K1L and E3L, followed by culture on the appropriate cells.

The F13L selection system described here relies on a different concept and takes advantage of the observation that F13L deficiency results in a severe block in virus transmission. By reintroduction of $F 13 \mathrm{~L}$, selection of virus recombinants only requires one series of blind passages, which can be monitored by cytopathic effect and red fluorescence, and can be done entirely in susceptible cells (BHK-21 or CEF). It is important to bear in mind that recombination of the vaccinia genome with a circular plasmid may result in single recombinants, with the transient integration of the whole plasmid. In this respect, the use of MVA $\triangle \mathrm{F} 13 \mathrm{~L}$ as the parental virus has the advantage that single recombinants can be easily recognized because of their red fluorescence, together with the large plaque phenotype.

The protocol presented here concludes with an almost homogeneous population of stable MVA recombinants containing a small proportion of single recombinants. The subsequent steps after applying selection may vary according to the use intended for the MVA recombinant. In some cases, the final population may be adequate to perform expression experiments. Alternatively, where homogeneity of the population is required, a final viruscloning step can be easily done from the virus stock after selection.

\section{ACKNOWLEDGMENTS}

This work was supported by contract QLK2-CT2002-01867 from the European Commission and grant no. BMC2002-03047 from Dirección General de Investigación Científica y Técnica, Spain to R.B.

\section{COMPETING INTERESTS STATEMENT}

\section{The authors declare no competing} interests.

\section{REFERENCES}

1. Stickl, H., V. Hochstein-Mintzel, A. Mayr, H.C. Huber, H. Schafer, and A. Holzner. 1974. MVA vaccination against smallpox: clinical tests with an attenuated live vaccinia virus strain (MVA) (author's transl). Dtsch. Med. Wochenschr. 99:2386-2392.

2.Hochstein-Mintzel, V., T. Hanichen, H.C. Huber, and H. Stickl. 1975. An attenuated strain of vaccinia virus (MVA). Successful intramuscular immunization against vaccinia and variola (author's transl). Zentralbl. Bakteriol. [Orig A] 230:283-297.

3. Moss, B., M.W. Carroll, L.S. Wyatt, J.R. Bennink, V.M. Hirsch, S. Goldstein, W.R. Elkins, T.R. Fuerst, et al. 1996. Host range restricted, non-replicating vaccinia virus vectors as vaccine candidates. Adv. Exp. Med. Biol. 397:7-13.

4. Sutter, G. and C. Staib. 2003. Vaccinia vectors as candidate vaccines: the development of modified vaccinia virus Ankara for antigen delivery. Curr. Drug Targets Infect. Disord. 3:263-271.

5.Carroll, M.W. and B. Moss. 1997. Host range and cytopathogenicity of the highly attenuated MVA strain of vaccinia virus: propagation and generation of recombinant viruses in a nonhuman mammalian cell line. Virology 238:198-211.

6. Drexler, I., K. Heller, B. Wahren, V. Erfle, and G. Sutter. 1998. Highly attenuated modified vaccinia virus Ankara replicates in baby hamster kidney cells, a potential host for virus propagation, but not in various human transformed and primary cells. J. Gen. Virol. 79:347-352.
7. Schneider, J., S.C. Gilbert, T.J. Blanchard, T. Hanke, K.J. Robson, C.M. Hannan, M. Becker, R. Sinden, et al. 1998. Enhanced immunogenicity for $\mathrm{CD} 8+\mathrm{T}$ cell induction and complete protective efficacy of malaria DNA vaccination by boosting with modified vaccinia virus Ankara. Nat. Med. 4:397-402.

8. Sutter, G., M. Ohlmann, and V. Erfle. 1995. Non-replicating vaccinia vector efficiently expresses bacteriophage T7 RNA polymerase. FEBS Lett. 371:9-12.

9.Staib, C., I. Drexler, M. Ohlmann, S. Wintersperger, V. Erfle, and G. Sutter. 2000. Transient host range selection for genetic engineering of modified vaccinia virus Ankara. BioTechniques 28:1137-1148.

10.Staib, C., M. Lowel, V. Erfle, and G. Sutter. 2003. Improved host range selection for recombinant modified vaccinia virus Ankara. BioTechniques 34:694-700.

11.Staib, C., I. Drexler, and G. Sutter. 2004 Construction and isolation of recombinant MVA. Methods Mol. Biol. 269:77-100.

12.Sutter, G., A. Ramsey Ewing, R. Rosales, and B. Moss. 1994. Stable expression of the vaccinia virus $\mathrm{K} 1 \mathrm{~L}$ gene in rabbit cells complements the host range defect of a vaccinia virus mutant. J. Virol. 68:4109-4116.

13.Hornemann, S., O. Harlin, C. Staib, S. Kisling, V. Erfle, B. Kaspers, G. Hacker, and G. Sutter. 2003. Replication of modified vaccinia virus Ankara in primary chicken embryo fibroblasts requires expression of the interferon resistance gene E3L. J. Virol. 77:8394-8407

14.Blasco, R. and B. Moss. 1995. Selection of recombinant vaccinia viruses on the basis of plaque formation. Gene 158:157-162.

15.Blasco, R. and B. Moss. 1991. Extracellular vaccinia virus formation and cell-to-cell virus transmission are prevented by deletion of the gene encoding the 37,000-dalton outer envelope protein. J. Virol. 65:5910-5920.

16.Blasco, R. and B. Moss. 1992. Role of cellassociated enveloped vaccinia virus in cell-tocell virus spread. J. Virol. 66:4170-4179.

17.Earl, P. and B. Moss. 1991. Expression of proteins in mammalian cells using vaccinia viral vectors. In F.M. Ausubel, R. Brent, R.E. Kingston, D.D. Moore, J.G. Seidman, J.A. Smith, and K. Struhl (Eds.), Current Protocols in Molecular Biology. Wiley-Interscience, New York.

18.Lorenzo, M.M., I. Galindo, and R. Blasco. 2004. Construction and isolation of recombinant vaccinia virus using genetic markers. Methods Mol. Biol. 269:15-30.

19.Sanchez-Puig, J.M., L. Sanchez, G. Roy, and R. Blasco. 2004. Susceptibility of different leukocyte cell types to Vaccinia virus infection. Virol. J. 1:10.

20.Barcena, J., M.M. Lorenzo, J.M. SanchezPuig, and R. Blasco. 2000. Sequence and analysis of a swinepox virus homologue of the vaccinia virus major envelope protein $\mathrm{P} 37$ (F13L). J. Gen. Virol. 81:1073-1085.

Received 12 April 2005; accepted 25 May 2005. 


\section{ShORT TeChNiCAL RePORTS}

Address correspondence to Rafael Blasco,

Dpt. Biotecnología, INIA, Ctra. La

Coruña km 7, E-28040 Madrid, Spain.

e-mail:blasco@inia.es

To purchase reprints

of this article, contact

Reprints@BioTechniques.com 